\title{
Über Elementarkettenbrüche, lineare Substitutionen und indefinite binäre quadratische Formen. I.
}

Von Herrn Paul Epstein in Straßburg i. E.

Den Ausgangspunkt für die vorliegende Arbeit bildete der Gedanke, die Lehre von den gewöhnlichen Kettenbrüchen auf die begrifflich einfachsten Elemente zurückzuführen, in ähnlicher Weise etwa, wie allgemeine lineare Substitutionen auf andere von besonders einfacher Natur zurückgeführt werden. Als Weg hierzu bot sich naturgemäß die Auflösung des Euklidischen Algorithmus in eine Reihe von elementaren Schritten, indem nämlich alle Teilquotienten $=1$ angenommen werden, oder, was auf dasselbe hinauskommt, die Ersetzung des Divisionsverfahrens durch eine Folge von Subtraktionen. So gelangt man zum Begriff der Zahlenkette, den wir an die Spitze unserer Darstellung gesetzt haben. Er führt unmittelbar zu einer besonderen Art von Kettenbrüchen - wir haben sie Elementarkettenbrüche (abgekürzt $\mathfrak{E} \Omega$.) genannt -, bei denen sämtliche Teilnenner +1 , alle Teilzähler entweder +1 oder -1 sind. Jede reelle Zahl läßt sich durch einen $\mathbb{\Omega}$. darstellen, und seine Näherungsbrüche sind die Haupt- und Nebennäherungsbrüche der gewöhnlichen Kettenbruchentwicklung*). Von den ER. gelangt man in einfachster Weise zu der Christoffel-Hurwitzschen Charakteristik, und sie gibt unmittelbar die Ver-

*) Dieselbe Eigenschaft haben die von Herrn Vahlen (Journ. f. Math. Bd. 115 S. 230) betrachteten "längsten Kettenbrüche" mit den Teilnennern 1 und 2. Die E̊. sind länger infolge des Auftretens der "überzähligen Näherungsbrüche“. Sie gehören auch nicht zu den "halbregelmäBigen Kettenbrüchen“. (Perron, Die Lehre von den Kettenbrüchen S. 154, Tietze, Math. Ann. Bd. 70.) 
wandlung des $\mathfrak{R}$. in einen gewöhnlichen Kettenbruch und umgekehrt. Damit wäre die ursprüngliche Aufgabe, die Theorie der gewöhnlichen Kettenbrüche auf möglichst einfacher Grundlage aufzubauen, erledigt. Aber darüber hinaus haben die ER. auch neben den gewöhnlichen Kettenbrüchen eine selbständige Bedeutung infolge des Umstandes, daß zwei reziproke Zahlen durchaus verschiedene Eన.-Entwicklungen besitzen. Dies hat weittragende Folgen für die Theorie der unimodularen ganzzahligen Substitutionen

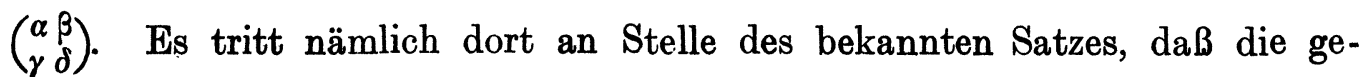
wöhnlichen Kettenbrüche für zwei äquivalente Zahlen von einer bestimm. ten Stelle an übereinstimmen, der Satz, daß die E尺.-Entwicklungen für zwei äquivalente Zahlen von einer bestimmten Stelle an entweder übereinstimmen oder zueinander reziprok sind (\$ 4, Satz 8). Dadurch offenbart sich eine Scheidung der linearen Substitutionen in zwei Arten, in ,gerade“ und „ungerade" Substitutionen, und diese Einteilung tritt vollständig gleichberechtigt neben die übliche Einteilung in ,eigentliche“ und ,uneigentliche" Substitutionen. So tritt neben die Gruppe $\mathfrak{A}$ der eigentlichen Substitutionen (Modulgruppe) die Gruppe $\mathfrak{B}$ der geraden Substitutionen, und zu ihnen kann man eine Gruppe $\mathfrak{E}$ fügen, die durch die eigentlichen geraden und die uneigentlichen ungeraden Substitutionen gebildet wird. Diese drei Gruppen sind isomorph, und*) es gibt in der Gruppe aller ganzzahligen unimodularen Substitutionen keine weitere Untergruppe vom Index 2. Sie enthalten als gemeinsame Untergruppe vom Index 2 die Gruppe der eigentlichen geraden Substitutionen**), von der wiederum die Hauptkongruenzgruppe 2. Stufe eine Untergruppe vom Index 3 darstellt. Wegen der Gleichberechtigung der Gruppen $\mathfrak{A}, \mathfrak{B}$ und $\mathfrak{E}$ muß es zu jeder mit der Modulgruppe zusammenhängenden Theorie eine entsprechende Theorie im Gebiete der Gruppe $\mathfrak{B}$ und der Gruppe $\mathfrak{C}$ geben. Dies wird in einer zweiten Abhandlung für die Reduktion der indefiniten binären quadratischen Formen innerhalb der Gruppe der geraden Substitutionen durchgeführt werden, und es werden sich dabei die Elementarkettenbrüche als das natürliche Hilfsmittel erweisen.

*) Auf diese (vermutlich nicht neue) Tatsache hat mich Herr J. Schur aufmerksam gemacht.

**) Diese Gruppe ist identisch mit der Untergruppe $\Gamma_{2}$ bei Klein-Fricke, Elliptische Modulfunktionen Bd. I, S. 288. 


\section{$\S 1$. Die Zahlenkette.}

Aus zwei gegebenen reellen Zahlen $a_{0}, a_{1}$ bilde man die Folge von positiven Zahlen:

(1.) $\quad a_{2}=\left|a_{0}-a_{1}\right|, a_{3}=\left|a_{1}-a_{2}\right|, a_{4}=\left|a_{2}-a_{3}\right|, \ldots$

Wir nennen dann $a_{0}, a_{1}, a_{2}, a_{3}, \ldots$ eine Kette von Zahlen und bezeichnen sie durch $\left[a_{0}, a_{1}\right]$.

Beispiel. Die Kette [28, 13] ist

$$
28,13,15,2,13,11,2,9,7,2,5,3,2,1,1,0,1,1,0,1, \ldots .
$$

Wenn, wie im vorliegenden Falle, zwei aufeinanderfolgende Zahlen einander gleich (allgemein $=d$ ) werden, so wiederholen sich von da ab die Zahlen $d, 0, d$ unbegrenzt, die Reihe wird periodisch. Nur in diesem Falle kann die Zahl 0 auftreten. Wir lassen gewöhnlich diesen periodischen Teil fort und nennen dann die Kette eine eigentliche abbrechende Kette. Nehmen wir aber eine gewisse Anzahl von Perioden hinzu, so haben wir eine überzählige abbrechende Kette. Wenn nichts anderes bemerkt ist, verstehen wir unter einer abbrechenden Kette immer eine eigentliche abbrechende Kette.

Sind $a_{0}$ und $a_{1}$ ganze Zahlen, so ist ihr größter gemeinsamer Teiler gleichzeitig Teiler von jeder Zahl der Kette, die Kette bricht ab und endigt mit dem größten gemeinsamen Teiler von $a_{0}$ und $a_{1}$. Sind $a_{0}, a_{1}$ relativ prim, so endigt die Kette immer mit den Zahlen 2, 1. Sie möge dann primitiv heißen.

Aus der Definition (1.) ist ersichtlich, daß jede Zahl der Kette in der Form

$$
\alpha a_{0}-\beta a_{1}
$$

mit ganzzahligen Koeffizienten $\alpha, \beta$ darstellbar ist. Es kann also die Zahl 0 nur auftreten, wenn das Verhältnis $\frac{a_{0}}{a_{1}}$ rational ist, und wir haben den Satz:

1. Die Kette $\left[a_{0}, a_{1}\right]$ bricht dann und nur dann $a b$, wenn $a_{0}$ und $a_{1}$ ein rationales Verhältnis haben.

Es seien nun $a_{0}, a_{1}$ ganze positive Zahlen ohne gemeinsamen Teiler. Wir teilen die abbrechende Kette $\left[a_{0}, a_{1}\right]$ in Abschnitte, sodaß in einem Abschnitt jede Zahl kleiner, als die vorhergehende ist; sobald eine Zahl größer 
ist, als die vorangehende, beginnt ein neuer Abschnitt. Jeder Abschnitt - allenfalls mit Ausnahme des ersten (wenn $a_{0}<a_{1}$ ist) - besteht aus mindestens zwei Zahlen, denn wenn mit der Zahl $a_{i}$ ein Abschnitt beginnt, so ist $a_{i-1}<a_{i}$, also $a_{i+1}=a_{i}-a_{i-1}<a_{i}$, d. h. $a_{i+1}$ gehört zum gleichen Abschnitt wie $a_{i}$. Auch der letzte Abschnitt einer eigentlichen Kette enthält mindestens die zwei Zahlen 2, 1, dagegen besteht bei einer überzähligen Kette der letzte Abschnitt nur aus der einen Zahl 1, der vorletzte Abschnitt endigt dann mit den Zahlen 1, 0. Es ist, wenn $a_{i-1}$ die Zahl am Schluß eines Abschnitts ist:

$$
a_{i+1}=a_{i}-a_{i-1}, \quad a_{i+2}=a_{i}-a_{i+1},
$$

also

$$
a_{i+2}=a_{i-1} \text {. }
$$

Die Zahlen am Schluß eines jeden Abschnitts wiederholen sich drei Stellen weiter.

Es möge so die Kette in Abschnitte von $\alpha, \beta, \gamma, \ldots \varrho, \sigma, \tau$ Zahlen zerfallen. Wir nennen dann

$$
\mid \alpha, \beta, \gamma, \ldots \rho, \sigma, \tau\}
$$

die Gliederung der Kette, und es sind darin sicher alle Zahlen mit Ausnahme der ersten und letzten $>1$. Unter dieser Bedingung kann aber jeder Komplex (3.) von ganzen positiven Zahlen als Gliederung einer bestimmten Kette aufgefaßt werden, denn es besteht der Satz:

2. Eine primitive Kette ist durch Angabe ihrer Gliederung vollständig bestimmt.

Zum Beweis brauchen wir nur das Verfahren anzugeben, um aus der Gliederung die Kette $\mathrm{zu}$ gewinnen, und schicken folgendes voraus: Es seien $u_{1}, u_{2}$ zwei positive Zahlen $\left(u_{1}<u_{2}\right)$. Eine endliche oder unendliche Folge von Zahlen

$$
u_{1}, u_{2}, u_{3}, u_{4}, \ldots
$$

bei der von $u_{3}$ ab jede Zahl die Summe der beiden vorhergehenden ist, heiße eine Fibonaccische Folge $\left(u_{1}, u_{2}\right)$.

Wir bilden nun, wenn $\tau>1$ ist, die Fibonaccische Folge $(1,2)$ mit $\tau$ Gliedern und schreiben sie von rechts nach links. Dann haben wir den letzten Abschnitt der gesuchten Kette. Aus den beiden letzten Gliedern $u_{\tau-1}, u_{\tau}$ berechnen wir 


$$
v_{1}=u_{\tau}-u_{\tau-1}, \quad v_{2}=u_{\tau}+v_{1}
$$

und bilden die Fibonaccische Folge $\left(v_{1}, v_{2}\right)$ mit $\sigma$ Gliedern. Aus ihren beiden letzten Gliedern berechnen wir

$$
w_{1}=v_{\sigma}-v_{\sigma-1}, \quad w_{2}=v_{\sigma}+w_{1}
$$

und bilden die Fibonaccische Folge $\left(w_{1}, w_{2}\right)$ mit $@$ Gliedern. So berechnet man zu jeder Zahl der vorgelegten Gliederung eine Fibonaccische Folge. Die Gesamtheit dieser Folgen, von rechts nach links geschrieben, bildet die gesuchte Kette, die wir mithin als die Kette $\{\alpha, \beta, \ldots \sigma, \tau\}$ bezeichnen können. Ist aber $\tau=1$, so besteht der letzte Abschnitt aus der einen Zahl 1, der vorletzte Abschnitt ist die Fibonaccische Folge $(0,1)$ mit $\sigma$ Gliedern, und die übrigen Abschnitte werden nach dem obigen Verfahren gefunden. Man erhält die überzählige Kette $\{\alpha, \beta, \gamma, \ldots \sigma, 1\}$.

Durch Weglassung der Perioden wird eine überzählige zu einer eigentlichen Kette. So wird, wenn $\sigma>\mathbf{3}$ ist, die überzählige Kette $\{\alpha, \beta, \ldots \varrho, \sigma, 1\}$ zur eigentlichen Kette $\{\alpha, \beta, \ldots \rho, \sigma-2\}$; wir schreiben

Ist aber $\sigma=2$, so ist

$$
\{\alpha, \beta, \ldots \varrho, \sigma, 1\}=\{\alpha, \beta, \ldots \varrho, \sigma-2\} \text {. }
$$

$$
\{\alpha, \beta, \ldots \varrho, 2,1\}=\{\alpha, \beta, \ldots \varrho\},
$$

ist $\sigma=3$, so ist $\{\alpha, \beta, \ldots \rho, \sigma-2\}$ wieder eine überzählige Kette und läßt sich weiter reduzieren.

Allgemein sei $\nu$ der letzte Abschnitt, der nicht $=3$ ist, und es sei $\nu>3$. Dann ist die überzählige Kette

$$
\{\alpha, \beta, \ldots \mu, \nu, 3,3, \ldots 3,1\}=\{\alpha, \beta, \ldots \mu, \nu-2\} \text {. }
$$

Ist aber $\nu=2$, so ist

$$
\{\alpha, \beta, \ldots \mu, 2,3,3, \ldots 3,1\}=\{\alpha, \beta, \ldots \mu\} \text {. }
$$

Beispiel. Aus der Gliederung

$$
\{2,2,3,3,4\}
$$

ergibt sich die Kette

$$
\begin{array}{ll|ll|lll|lll|llll}
28 & 13 & 15 & 2 & 13 & 11 & 2 & 9 & 7 & 2 & 5 & 3 & 2 & 1 .
\end{array}
$$

Wir leiten nun aus einer Kette eine neue Kette ab, indem wir den letzten Abschnitt um eine Zahl verkürzen. Aus der Kette $\{\alpha, \beta, \ldots \sigma, \tau\}$ entsteht so die erste abgeleitete Kette $\{\alpha, \beta, . . \sigma, \tau-1\}$. Im vorliegenden Beispiel ist dies die Kette $\{2,2,3,3,3\}$ :

$$
\begin{array}{ll|ll|lll|lll|lll}
15 & 7 & 8 & 1 & 7 & 6 & 1 & 5 & 4 & 1 & 3 & 2 & 1 ;
\end{array}
$$


aus ihr entsteht die zweite abgeleitete Kette $\{2,2,3,3,2\}$ :

$$
\begin{array}{ll|ll|lll|lll|ll}
13 & 6 & 7 & 1 & 6 & 5 & 1 & 4 & 3 & 1 & 2 & 1 .
\end{array}
$$

Die nächste abgeleitete Kette $\{2,2,3,3,1\}$ jst eine überzählige Kette:

$$
\begin{array}{ll|ll|lll|lll|l}
2 & 1 & 1 & 0 & 1 & 1 & 0 & 1 & 1 & 0 & 1,
\end{array}
$$

und sie reduziert sich, wie man sieht, übereinstimmend mit (5.) auf $\{2\}$, d. i. die einfache Kette 2,1. Wir behalten aber die überzählige Kette bei und können aus ihr wieder eine $\operatorname{Kette}^{\text {Ta }}\{2,2,3,3\}$ ableiten, und so fortfahrend erhalten wir schließlich aus der zugrunde gelegten Kette

\begin{tabular}{|c|c|c|c|c|c|c|}
\hline 28 & 13 & 152 & 13 & 112 & 972 & $\begin{array}{llll}5 & 3 & 2 & 1\end{array}$ \\
\hline 15 & 7 & 81 & 7 & 61 & $\begin{array}{lll}5 & 4 & 1\end{array}$ & $\begin{array}{lll}3 & 2 & 1\end{array}$ \\
\hline 13 & 6 & 71 & 6 & 51 & 431 & 21 \\
\hline 2 & 1 & 10 & 1 & 10 & 110 & 1 \\
\hline 11 & 5 & 61 & 5 & 41 & 321 & \\
\hline 9 & 4 & 51 & 4 & 31 & 21 & \\
\hline 2 & 1 & 10 & 1 & 10 & 1 & \\
\hline 7 & 3 & 41 & 3 & 21 & & \\
\hline 5 & 2 & 31 & 2 & 1 & & \\
\hline 2 & 1 & 10 & 1 & & & \\
\hline 3 & 1 & 21 & & & & \\
\hline 1 & 0 & 1 & & & & \\
\hline 2 & 1 & & & & & \\
\hline 1 & & & & & & \\
\hline
\end{tabular}
$\{2,2,3,3,4\}$ eine vollständige Tafel abgeleiteter Ketten:

Diese Tafel hat bemerkenswerte Eigenschaften. Es ist nicht nur jede Horizontalreihe, sondern auch jede Vertikalreihe eine Kette, genauer: Von jeder Stelle der Tafel geht nach rechts und nach unten eine Kette, und es hat die vertikale Kette die umgekehrte Gliederung wie die horizontale Kette. In unserem Beispiel hat die erste vertikale Kette die Gliederung $\{4,3,3,2,2\}$; sie möge die erste Adjungierte der vorgelegten Kette heißen. Die darauf folgenden Vertikalreihen bilden die zweite, dritte,... Adjungierte. Jede Adjungierte ist die abgeleitete Kette der vorhergehenden. Ist $\left[a_{0}, a_{1}\right]$ die gegebene, $\left[b_{0}, b_{1}\right]$ die erste abgeleitete Kette, so ist $\left[a_{0}, b_{0}\right]$ die erste, $\left[a_{1}, b_{1}\right]$ die zweite Adjungierte. Umgekehrt ist die gegebene Kette die erste Adjungierte ihrer Adjungierten. 
Da die Tafel auch für die erste Adjungierte eine vollständige Tafel abgeleiteter Ketten darstellt, so zerfällt sie in vertikale und horizontale Abschnitte. Jede Kette am Ende eines Abschnitts ist überzählig und stimmt mit der drittfolgenden Kette überein. Dies folgt unmittelbar aus Formel (2).

Sind

$$
\begin{array}{lll}
a_{i-1} & a_{i} & a_{i+1} \\
b_{i-1} & b_{i} & b_{i+1} \\
c_{i-1} & c_{i} & c_{i+1}
\end{array}
$$

9 Zahlen in je 3 aufeinanderfolgenden Horizontal- und Vertikalreihen, so sind alle aus der Matrix dieser Zahlen gebildeten Unterdeterminanten 2. Grades gleich \pm 1 und die Determinante aus den 9 Zahlen ist Null.

Man hat hiernach ein einfaches Verfahren zur Auflösung der Diophantischen Gleichungen

$$
a x-b y= \pm 1
$$

Man bilde die Kette $[a, b]$ und ihre abgeleitete Kette. Dann liefern die beiden ersten Zahlen der abgeleiteten Kette Lösungen $y, x$ der einen Gleichung, und es sind $b-x$ und $a-y$ Lösungen der andern Gleichung.

Diese Eigenschaften der vollständigen Tafel der abgeleiteten Ketten werden evident, wenn wir nun dazu übergehen, den Zusammenhang der Zahlenketten mit den Kettenbrüchen zu betrachten.

\section{§ 2. Endliche Elementarkettenbrüche.}

Die Bildung der Zahlenkette ist ein besonderer Euklidischer Algorithmus, bei dem alle Teilquotienten =1 sind, und zwar für die Kette mit der Gliederung $\{\alpha, \beta, \gamma, \ldots\}$ :

$$
\begin{gathered}
a_{0}=1 \cdot a_{1}+a_{2} \\
a_{1}=1 \cdot a_{2}+a_{3} \\
\vdots \\
\hline a_{a-1}=1 \cdot a_{a}-a_{\alpha+1} \\
a_{a}=1 \cdot a_{a+1}+a_{a+2} \\
\vdots \\
\hline a_{a+\beta-1}=1 \cdot a_{a+\beta}-a_{a+\beta+1} \\
a_{a+\beta}=1 \cdot a_{a+\beta+1}+a_{a+\beta+2} \\
\vdots
\end{gathered}
$$


Hieraus entspringt eine Kettenbruchentwicklung

$\frac{a_{0}}{a_{1}}=\underbrace{1+\frac{1}{\mid 1}+\frac{1}{\mid 1}+\cdots+\frac{1}{\mid 1}}_{a}-\underbrace{\frac{1}{\mid 1}+\frac{1}{\mid 1}+\cdots+\frac{1}{\mid 1}}_{\beta}-\underbrace{\frac{1}{\mid 1}+\frac{1}{\mid 1}+\cdots+\frac{1}{\mid 1}}_{\gamma}-\cdots$,

also ein Kettenbruch, der ebensoviele Teilnenner, sämtlich vom Werte +1 , besitzt, als die Anzahl der Zahlen in der Kette beträgt, und bei dem die ersten Teilzähler in jedem Abschnitt (vom zweiten Abschnitt an) gleich -1 , alle übrigen Teilzähler gleich +1 sind. Einen solchen Kettenbruch nennen wir Elementarkettenbruch (abgekürzt \&̊.) und können ihn vollkommen verständlich durch die Gliederung der Kette bezeichnen, indem wir ihn schreiben:

$$
\text { E }\{\alpha, \beta, \gamma, \ldots \sigma, \boldsymbol{x}\} \text {. }
$$

$\{\alpha, \beta, \gamma, \ldots \sigma, \tau\}$ nennen wir dann auch die Gliederung des Kettenbruchs, $\alpha, \beta, \gamma, \ldots$ heißen die Abschnitte des Kettenbruchs. Es besteht also der Satz:

1. Zu jeder Kette $\left[a_{0}, a_{1}\right]$ gehört ein Elementarkettenbruch von der gleichen Gliederung, und sein Wert ist $\frac{a_{0}}{a_{1}}$.

So gehört zu der Kette $[28,13]$ der $\mathfrak{R}$.:

$$
\text { E }\{2,2,3,3,4\}
$$

oder

$$
\frac{28}{13}=1+\frac{1}{\mid 1}-\frac{1}{\mid 1}+\frac{1}{\mid 1}-\frac{1}{\mid 1}+\frac{1}{\mid 1}+\frac{1}{\mid 1}-\frac{1}{\mid 1}+\frac{1}{\mid 1}+\frac{1}{\mid 1}-\frac{1}{\mid 1}+\frac{1}{\mid 1}+\frac{1}{\mid 1}+\frac{1}{\mid 1} .
$$

Jeder Abschnitt eines $\mathfrak{A}$. außer dem ersten und letzten besteht aus mindestens zwei Gliedern, d. h. es folgen niemals zwei Teilzähler - 1 aufeinander, und umgekehrt ist jeder derartige Kettenbruch (mit lauter Teilnennern +1 und Teilzählern \pm 1$)$ ein $\mathfrak{R}$. und einer bestimmten Kette zugeordnet. Zur Darstellung von negativen Zahlen werden wir auch $\mathfrak{\text { l. }}$ zulassen, bei denen die beiden ersten Abschnitte gleich 1 sind (s. u. Formel (14.)).

$\mathrm{Zu}$ einer eigentlichen Kette gehört ein eigentlicher $\mathfrak{\Re . ; ~ b e i ~ i h m ~ i s t ~}$ der letzte Abschnitt $\tau>1$, der Kettenbruch schließt mit $\frac{+1}{1}$. Einer überzähligen Kette entspricht ein überzähliger હミ. mit $\tau=1$; er schließt mit $\frac{-1}{1}$. 
Den abgeleiteten Ketten entsprechen (in umgekehrter Reihenfolge) die Näherungsbrüche des $\mathbb{E} \Omega$. Es sind dies die Elementarkettenbrüche (wir lassen das Zeichen $\&$ weg):

$$
\begin{array}{rc}
\{1\} ; & \{2\} ; \ldots\{\alpha\} \\
\{\alpha, 1\} ; & \{\alpha, 2\} ; \ldots\{\alpha, \beta\} \\
\{\alpha, \beta, 1\} ; & \{\alpha, \beta, 2\} ; \ldots\{\alpha, \beta, \gamma\} \\
& \vdots \\
\{\alpha, \beta, \ldots \sigma, 1\} ;\{\alpha, \beta, \ldots \sigma, 2\} ; \ldots & \{\alpha, \beta, \ldots \sigma, \tau\} .
\end{array}
$$

Die Näherungsbrüche am Beginn eines jeden Abschnitts (vom zweiten Ab. schnitt an) sind überzählig; jeder von ihnen ist gleich dem um 3 Stellen zurückliegenden Näherungsbruch. Die übrigen Näherungsbrüche sind die eigentlichen Näherungsbrüche. Bei dem obigen 토. hat man die Näherungsbrüche

$$
\frac{1}{1}, \frac{2}{1}\left|\frac{1}{0}, \frac{3}{1}\right| \frac{2}{1}, \frac{5}{2}, \frac{7}{3}\left|\frac{2}{1}, \frac{9}{4}, \frac{11}{5}\right| \frac{2}{1}, \frac{13}{6}, \frac{15}{7}, \frac{28}{13}
$$

Hier wird auch der Näherungsbruch $\frac{1}{0}$ als überzählig angesehen, indem man dem Näherungsbruch $\frac{1}{1}$ den Bruch $\frac{1}{0}$ als nullten eigentlichen Näherungsbruch vorausschickt. Für die Berechnung der Näherungsbrüche besteht das Gesetz:

Der Zähler (Nenner) eines eigentlichen Näherungsbruches ist die Summe
Differenz der beiden vorhergehenden Zähler (Nenner).

Um die Gesamtheit der Näherungsbrüche einfach zu übersehen, verstehen wir unter einer gebrochenen Fibonaccischen Folge von $n$ Gliedern eine Reihe von $n$ Brüchen $\frac{a_{1}}{b_{1}}, \frac{a_{2}}{b_{2}}, \frac{a_{3}}{b_{3}}, \ldots$, deren Zähler und Nenner Fibonaccische Folgen sind, sodaß also $\frac{a_{i+1}}{b_{i+1}}=\frac{a_{i}+a_{i-1}}{b_{i}+b_{i-1}}$ ist, und bezeichnen eine solche gebrochene Folge durch

$$
\frac{\left(a_{1}, a_{2}\right)}{\left(b_{1}, b_{2}\right)_{n}}
$$

Es sei nun zunächst $\alpha>1$. Dann bilden die $\alpha$ ersten Näherungsbrüche die gebrochene Folge $\frac{(1,2)}{(1,1)_{a}}$. Die beiden letzten Brüche seien $\frac{a_{a-1}}{a_{a-1}^{\prime}}, \frac{a_{a}}{a_{\alpha}^{\prime}} ;$ man berechnet 


$$
\begin{array}{ll}
b_{1}=a_{\alpha}-a_{\alpha-1}, & b_{2}=a_{a}+b_{1} \\
b_{1}^{\prime}=a_{a}^{\prime}-a_{a-1}^{\prime}, & b_{2}^{\prime}=a_{a}^{\prime}+b_{1}^{\prime}
\end{array}
$$

und hat in der gebrochenen Folge $\frac{\left(b_{1}, b_{2}\right)}{\left(b_{1}^{\prime}, b_{2}^{\prime}\right)_{\beta}}$ die $\beta$ Näherungsbrüche des zweiten Abschnitts. Die beiden letzten Näherungsbrüche liefern

$$
\begin{array}{ll}
c_{1}=b_{\beta}-b_{\beta-1}, & c_{2}=b_{\beta}+c_{1} \\
c_{1}^{\prime}=b_{\beta}^{\prime}-b_{\beta-1}^{\prime}, & c_{2}^{\prime}=b_{\beta}^{\prime}+c_{1}^{\prime},
\end{array}
$$

und die gebrochene Folge $\frac{\left(c_{1}, c_{2}\right)}{\left(c_{1}^{\prime}, c_{2}^{\prime}\right)_{\gamma}}$ gibt die $\gamma$ Näherungsbrüche des dritten Abschnitts. So erhält man der Reihe nach zu jedem Abschnitt des $\mathfrak{E} \Omega$. die zugehörigen Näherungsbrüche.

Ist aber $\alpha=1$, so ist $\frac{1}{1}$ der erste Näherungsbruch; die $\beta$ Näherungsbrüche des zweiten Abschnitts bilden die gebrochene Folge $\frac{(0,1)}{(1,2)_{\beta}}$, und dann rechnet man wie oben weiter.

Aus der Bildungsweise der Näherungsbrüche erkennt man sofort:

2. Die Zähler und Nenner sämtlicher Näherungsbrüche bilden (von rück wärts genommen) Zahlenketten von der umgekehrten Gliederung, wie der ㅌ.. und sein erster vollständiger Quotient, d. h. es sind die Ketten

$$
\{\tau, \sigma, \ldots \beta, \alpha\} \text { und }\{\tau, \sigma, \ldots \beta, \alpha-1\} \text {. }
$$

Sie bilden mithin die erste und zweite Adjungierte der dem Є尺. zugeordneten Zahlenkette $\left[a_{0}, a_{1}\right]$.

Ist $\left[b_{0}, b_{1}\right]$ die erste abgeleitete Kette von $\left[a_{0}, a_{1}\right]$, so kann man die Gesamtheit der Näherungsbrüche symbolisch durch den Ausdruck

zusammenfassen, und es ist

$$
\frac{\left[a_{0}, b_{0}\right]}{\left[a_{1}, b_{1}\right]}
$$

$$
\begin{aligned}
& \frac{a_{0}}{a_{1}}=\Subset\{\alpha, \beta, \ldots \sigma, \tau\} \\
& \frac{b_{0}}{\bar{b}_{1}}=\mathfrak{E}\{\alpha, \beta, \ldots \sigma, \tau-1\} \\
& \frac{a_{0}}{b_{0}}=\Subset\{\tau, \sigma, \ldots \beta, \alpha\} \\
& \frac{a_{1}}{b_{1}}=\Subset\{\tau, \sigma, \ldots \beta, \alpha-1\} .
\end{aligned}
$$


Die Anzahl der Glieder einer abbrechenden Kette, also auch die Anzahl der Teilnenner oder der Näherungsbrüche des zugehörigen $€ \Re$. nennen wir die Länge der Kette und des Kettenbruchs. Sie ist

$$
\alpha+\beta+\gamma+\cdots+\sigma+\tau=l \text {. }
$$

Ferner möge die um 1 verminderte Anzahl der Abschnitte oder die Anzahl der negativen Teilzähler oder der überzähligen Näherungsbrüche als die Ordnung der Kette und des Kettenbruchs bezeichnet werden.

Ebenso, wie man einen überzähligen $\mathfrak{E} \Omega$. auf einen eigentlichen reduzieren kann, kann man auch einen eigentlichen $\mathfrak{E} \Omega$. zu einem überzähligen von beliebiger Länge erweitern, und zwar auf zwei Arten. Nach (4.) und (5.) in $\S 1$ ist:

$$
\mathfrak{E}\{\alpha, \beta, \ldots \nu\}=\mathbb{E}\{\alpha, \beta, \ldots \mu, \nu+2,3,3, \ldots 3,1\}
$$

und

(4.) $\mathbb{E}\{\alpha, \beta, \ldots \nu\}=\mathbb{E}\{\alpha, \beta, \ldots \mu, \nu, 2,3,3, \ldots 3,1\}$.

In der ersten Formel kann der ㅌ․ links auch ein überzähliger sein.

Es sei $\frac{P_{\boldsymbol{i}}}{\bar{Q}_{i}} \operatorname{der} i^{\text {to }}$ Näherungsbruch, und es werde

$$
P_{i} Q_{i-1}-P_{i-1} Q_{i}=\partial_{i}
$$

gesetzt. Ist nun $\frac{P_{i}}{Q_{i}}$ ein eigentlicher Näherungsbruch, so ist $P_{i}=P_{i-1}+P_{i-2}$, $Q_{i}=Q_{i-1}+Q_{i-2}$, also

$$
\partial_{i}=-\partial_{i-1}
$$

Ist aber $\frac{P_{i}}{Q_{i}}$ ein überzähliger Näherungsbruch, so ist $P_{i}=P_{i-1}-P_{i-2}$, $Q_{i}=Q_{i-1}-Q_{i-2}$, also

$$
\partial_{i}=\partial_{i-1}
$$

Da nun $\partial_{1}=-1$ ist, so folgt:

3. Die zu den Näherungsbrüchen gehörenden Zahlen $\partial_{i}$ sind in einem Abschnitt abwechselnd +1 und -1 . Die erste Zahl in jedem Abschnitt stimmt mit der letzten des vorausgehenden Abschnitts überein. Die erste von allen Zahlen $\partial_{i}$ ist -1 .

Der Näherungsbruch $\frac{P_{i}}{Q_{i}}$ hat als ES. die Länge $i$; seine Ordnung sei $m$, er gehöre also zum $(m+1)^{\text {ten }}$ Abschnitt. Dann ist offenbar

$$
\partial_{i}=(-1)^{i-m} \text {. }
$$

Führt man in (5.): $P_{i-1}=P_{i} \mp P_{i-2}, Q_{i-1}=Q_{i} \mp Q_{i-2}$ ein, je nachdem 
$\frac{P_{i}}{Q_{i}}$ ein eigentlicher oder ein überzähliger Näherungsbruch ist, so folgt:

4. Es ist

(7.) $\quad P_{i} Q_{i-8}-P_{i-2} Q_{i}=(-1)^{i-m+1}$ oder $(-1)^{i-m}$,

je nachdem $\frac{P_{i}}{Q_{i}}$ ein eigentlicher oder ein überzähliger Näherungsbruch ist.

Ist $\frac{P_{i}}{\bar{Q}_{i}}$ nicht der letzte Näherungsbruch in einem Abschnitt, also etwa, wenn der Abschnitt $\mu=\mu_{1}+\mu_{2}$ ist:

$$
\frac{P_{i}}{Q_{i}}=\mathbb{E}\left\{\alpha, \beta, \gamma, \ldots \mu_{1}\right\},
$$

und ist $x_{i}$ der zugehörige vollständige Quotient:

so ist

$$
x_{i}=\Subset\left\{\mu_{2}, \nu, \ldots \sigma, \tau\right\},
$$

$$
\notin\{\alpha, \beta, \gamma, \ldots \sigma, \tau\}=\frac{x_{i} P_{i}+P_{i-1}}{x_{i} Q_{i}+Q_{i-1}} .
$$

Ist aber $\frac{P_{i}}{Q_{i}}$ der letzte Näherungsbruch in einem Abschnitt, also

$$
\frac{P_{i}}{Q_{i}}=\mathfrak{E}\{\alpha, \beta, \ldots \mu\} ; x_{i}=\mathbb{E}\{\nu, \ldots \sigma, \tau\},
$$

so ist

$$
\mathfrak{E}\{\alpha, \beta, \ldots \sigma, \tau\}=\frac{x_{i} P_{i}-P_{i-1}}{x_{i} Q_{i}-Q_{i-1}}
$$

Es sei $a$ der Wert des ER. und $\frac{P_{i}}{\bar{Q}_{i}}$ nicht der letzte Näherungsbruch in einem Abschnitt. Dann ist

$$
\begin{gathered}
a-\frac{P_{i}}{Q_{i}}=\frac{x_{i} P_{i}+P_{i-1}}{x_{i} Q_{i}+Q_{i-1}}-\frac{P_{i}}{Q_{i}}=-\frac{\partial_{i}}{Q_{i}\left(x_{i} Q_{i}+Q_{i-1}\right)}, \text { also } \\
\left|a-\frac{P_{i}}{Q_{i}}\right|=\frac{1}{Q_{i}\left(x_{i} Q_{i}+Q_{i-1}\right)} .
\end{gathered}
$$

Der vorausgehende Näherungsbruch gehöre zum gleichen Abschnitt; der zugehörige vollständige Quotient ist $x_{i-1}=1+\frac{1}{x_{i}}$, und es wird

Setzt man

$$
\begin{gathered}
\left|a-\frac{P_{i-1}}{Q_{i-1}}\right|=\frac{1}{Q_{i-1}\left(x_{i-1} Q_{i-1}+Q_{i-2}\right)}=\frac{x_{i}}{Q_{i-1}\left[x_{i}\left(Q_{i-1}+Q_{i-2}\right)+Q_{i-1}\right]} \\
=\frac{x_{i}}{Q_{i-1}\left(x_{i} Q_{i}+Q_{i-1}\right)}=\frac{x_{i} Q_{i}}{Q_{i-1}}\left|a-\frac{P_{i}}{Q_{i}}\right| .
\end{gathered}
$$




$$
\left|a Q_{i}-P_{i}\right|=\Delta_{i}
$$

so ist also

(10.)

$$
\Delta_{i-1}=x_{i} \Delta_{i}
$$

Man sieht leicht, daß diese Formel allgemein gilt, auch wenn $\frac{P_{i}}{Q_{i}}$ der letzte Näherungsbruch in einem Abschnitt oder ein überzähliger Näherungsbruch ist. Nun ist $x_{i}=\frac{1}{\left|x_{i-1}-1\right|}$, also

und damit hat man den Satz:

$$
\Delta_{i}=\left|\Delta_{i-2}-\Delta_{i-1}\right| \text {, }
$$

5. Die Differenzen $\Delta_{i}=\left|a Q_{i}-P_{i}\right|$ bilden eine Zahlenkette, die mit den Zahlen $\Delta_{0}=1, \Delta_{1}=|a-1|$ beginnt. Ihre Gliederung ist $\{\alpha-1, \beta, \gamma, \ldots \tau\}$ oder $\{\beta, \gamma, \ldots \tau\}$, je nachdem $\alpha \geqq 1$ ist.

Der $\mathfrak{E} \Omega$. für $a=\frac{a_{0}}{a_{1}}$ entspringt aus der Zahlenkette $\left[a_{0}, a_{1}\right]$, und nach dem eben gefundenen Satz ist

$$
\left|a_{0} Q_{i}-a_{1} P_{i}\right|=a_{i+1} \text {. }
$$

Hiermit sind die Glieder der Zahlenkette durch die beiden ersten Glieder ausgedrückt.

Das Vorzeichen der oben bestimmten Größe $\partial_{i}=P_{i} Q_{i-1}-P_{i-1} Q_{i}= \pm 1$

wollen wir den Index des Näherungsbruches $\frac{P_{i}}{Q_{i}}$ nennen. Wir lassen nun die Indices der überzähligen Näherungsbrüche fort, behalten aber die Einteilung in Abschnitte bei und nennen die so gebildete Gesamtheit der Indices die Indexkette des $\mathfrak{S}$. Es besteht also die Indexkette zum Kettenbruch $\&\{\alpha, \beta, \gamma, \ldots \tau\}$ aus lauter abwechselnden Vorzeichen \pm , mit beginnend, und zwar im ersten Abschnitt $\alpha$, in den folgenden Abschnitten $\beta-1, \gamma-1, \ldots \tau-1$ Indices. Durch die Indexkette ist der $€ \Re$. vollständig bestimmt. So gehört zur Indexkette

$-+-1+-+-+|-|+|-|+-1+-+|-|+-+-1+-1+-1+$ $\operatorname{der} \mathfrak{E}$.: $\mathbb{E}\{3,6,2,2,2,3,4,2,5,3,3,2\}$.

Einer gegebenen Indexkette kann man eine zweite Kette zuordnen, indem man die fehlenden Trennungsstriche zwischen je zwei Indices hinzufügt und dafür die vorhandenen Trennungsstriche wegläßt. Diese neue Indexkette heiße die zur ersten reziproke Indexkette.

Die zur obigen Kette reziproke Kette ist also: 
$-1+|-+|-|+|-|+-+-+|-+|-|+-+|-|+|-+|-+1-+$ und der zugehörige $\mathfrak{A}$.: $\mathfrak{E}\{1,2,3,2,2,2,6,3,2,4,2,2,3,3,3\}$.

Die Indexkette steht in einfachem Zusammenhang mit der von Christoffel und Herrn Hurwitz*) eingeführten Charakteristik. Wir stellen zunächst fest, daß die Näherungsbrüche des E્న. auf Grund der oben beschriebenen Bildungsweise dieselben sind, wie die, welche man nach $H u r$. witz durch die Fareyschen Reihen**) erhält. Man gibt nun jedem Näherungsbruch den Charakter + oder - , je nachdem er größer oder kleiner ist, als der Wert $a$ des Kettenbruchs. Die Charaktere der eigentlichen Näherungsbrüche bilden in ihrer Gesamtheit die Charakteristik von $a$.

Ist $\frac{P_{i}}{Q_{i}}$ nicht der letzte Näherungsbruch in einem Abschnitt, so ist $a=\frac{P_{i} x+P_{i-1}}{Q_{i} x+Q_{i-1}} \lessgtr \frac{P_{i}}{Q_{i}}$, je nachdem $P_{i} Q_{i-1}-P_{i-1} Q_{i} \gtrless 0$ ist, also je nach$\operatorname{dem} \frac{P_{i}}{Q_{i}}$ positiven oder negativen Index hat. Ist aber $\frac{P_{i}}{Q_{i}}$ der letzte Näherungsbruch in einem Abschnitt, so ist $a=\frac{P_{i} x-P_{i-1}}{Q_{i} x-Q_{i-1}} \lessgtr \frac{P_{i}}{Q_{i}}$, je nachdem der Index von $\frac{P_{\boldsymbol{i}}}{Q_{i}}$ negativ oder positiv ist. Es besteht also der Satz:

6. Für alle Näherungsbrüche in einem Abschnitt mit Ausnahme des letzten stimmt der Charakter mit dem Index überein; für den letzten Näherungsbruch in jedem Abschnitt ist der Charakter dem Index entgegengesetat.

Es wird also die Indexkette in die Charakteristik verwandelt, indem man nur jeden letzten Index in einem Abschnitt durch den entgegengesetzten ersetzt.

Von der Charakteristik gelangt man bekanntlich sofort zu der gewöhnlichen Kettenbruchentwicklung der Zahl $a$. Seien nämlich $q, q_{1}, q_{2}, \ldots$

*) Christoffel, Lehrsätze über arithmetische Eigenschaften der Irrationalzahlen, Annali di Matematica (2) 15 (1888).

Hurwitz, Uber die angenäherte Darstellung der Zahlen durch rationale Brüche. Math. Ann. 44 (1894).

${ }^{* *}$ ) Es ist gewiß bemerkenswert, daß bereits Nicolas Chuquet in seiner Triparty, 1484 in derselben Weise die Näherungsbrüche einer gegebenen Größe bildet. Vgl. Cantor, Gesch. d. Math. Bd. 2, S. 352. Wertheim, Zeitschr. f. Math. u. Phys. Bd. 42 (1898) Suppl. S. 149. 
die Anzahlen der aufeinander folgenden gleichen Charaktere, beginnend mit $q$ negativen Charakteren, so ist*)

$$
a=\left[q, q_{1}, q_{2}, \ldots\right]=q+\frac{1}{q_{1}}+\frac{1}{q_{2}}+\ldots
$$

Beginnt aber die Charakteristik mit $q$ positiven Charakteren, so ist

$$
a=\left[0, q, q_{1}, q_{2}, \ldots\right]=\frac{1}{q}+\frac{1}{q_{1}}+\ldots
$$

Indem wir die Indexkette in die Charakteristik verwandeln, haben wir

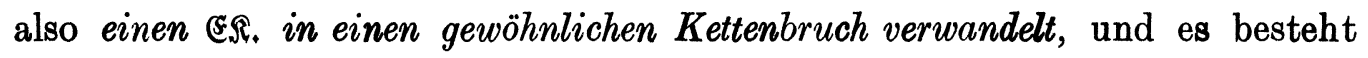
der Satz:

7. Die eigentlichen Näherungsbrüche des Elementarkettenbruchs für eine Zahl a stimmen überein mit den Haupt- und Näherungsbrüchen**) der gewöhnlichen Kettenbruchentwicklung von a.

Ist wieder $l$ die Länge, $m$ die Ordnung des $\mathfrak{E} \Omega$., so ist also die Summe aller Teilquotienten des gewöhnlichen Kettenbruchs

$$
q+q_{1}+q_{2}+\cdots+q_{n}=l-m,
$$

was sich auch daraus ergibt, daß Indexreihe und Charakteristik gleich lang sind.

So erhält man nach Satz 6. aus der Indexkette $-+-1+-+-+|-|+|-|+-|+-+|-\mid+-+-1+-1+-1+$ die Charakteristik

$$
-+++-+--+-+++--++-+++++ \pm
$$

und der zugehörige $\mathfrak{E} \Re$. ist gleich dem regelmäßigen Kettenbruch

$$
[1,3,1,1,2,1,1,4,2,2,1,7] \text {. }
$$

Der letzte Charakter kann nach Belieben + oder - genommen werden, entsprechend dem Umstand, daß man den Kettenbruch anstatt mit $q_{n}(>1)$ auch mit $q_{n}-1+\frac{1}{1}$ schließen kann.

*) Der kleine formale Unterschied gegen die Darstellung von Herrn Hurwite (a. a. O. S. 430) kommt nur daher, daß dort dem Näherungsbruch $\frac{1}{1}$ noch die Brüche $\frac{1}{0}, \frac{0}{1}$ vorangehen.

**) Dabei rechnen wir abweichend von Perron, Die Lehre von den Kettenbrüchen S. 55, die Zahlen $\frac{1}{1}, \frac{2}{1}, \ldots \frac{q-1}{1}$ zu den Näherungsbrtichen des Kettenbruchs. 
Ebenso einfach ist die Verwandlung eines gewöhnlichen Kettenbruchs in einen Elementarkettenbruch. Man schreibt unter die Charakteristik des Kettenbruchs eine gleichlange Reihe von abwechselnden Vorzeichen \pm , mit - beginnend. So oft ein Vorzeichen von dem darüberstehenden Charakter verschieden ist, ist ein Abschnitt zu Ende. Damit hat man die Indexkette des Eన. und diesen selbst. So hat der gewöhnliche Kettenbruch $[0,3,2,1,5]$ die Charakteristik

also ist die Indexkette

$$
+++-+----
$$

$$
-1+-1+1-+-+1-+1-
$$

und der ER.: E $\{1,3,2,5,3,2\}$.

Beim Ubergang von der Indexkette zur reziproken Kette wird jeder innere (d. h. nicht einen Abschnitt schließende) Index zu einem letzten und jeder letzte Index zu einem inneren. Es hat daher die zur reziproken Kette gehörige Charakteristik gerade die entgegengesetzten Charaktere, wie die erste Charakteristik, folglich sind die entsprechenden Kettenbrüche zueinander reziprok; es besteht der Satz:

8. Die zu reziproken Indexketten gehörenden Elementarkettenbrüche sind reziprok.

Die explizite Darstellung des zum ES. E $\{\alpha, \beta, \ldots \tau\}$ reziproken EN. ist nicht eben einfach und kann hier übergangen werden*).

Dagegen bestehen einfache Beziehungen zwischen den Längen $l, l^{\prime}$ und den Ordnungen $m, m^{\prime}$ von zwei reziproken Elementarkettenbrüchen. Zunächst sind die beiden reziproken Indexketten gleich lang, also

$$
l-m=l^{\prime}-m^{\prime} \text {. }
$$

Denkt man sich die beiden Ketten aufeinandergelegt, so fallen die Trennungsstriche der einen Kette zwischen die nicht getrennten Indices der an. dern, also ist ihre Anzahl in beiden Ketten zusammen:

$$
m+m^{\prime}=l-m-1 \text {. }
$$

Hiernach ist die Ordnung des reziproken $\mathbb{R}$.

$$
m^{\prime}=l-2 m-1
$$

und die Länge

*) Es sei hierfür auf eine demnăchst in der Mathematischen Zeitschrift erscheinende Arbeit „¿'ber Möbiuskettenbrüche und Elementarkettenbrüche" verwiesen. 
$\left(13^{\circ}.\right)$

$$
l^{\prime}=2 l-3 m-1 .
$$

Bis jetzt haben wir die durch einen $\mathbb{E} \Omega$. dargestellte Zahl als positiv angenommen. Wir können aber auch eine negative rationale $\mathrm{Zahl}$ in einen ङన. entwickeln, und zwar auf zwei Arten. Seien $a_{0}$ und $a_{1}$ positive ganze Zahlen, so können wir entweder $-\frac{a_{0}}{a_{1}}=\frac{-a_{0}}{a_{1}}$ oder $=\frac{a_{0}}{-a_{1}}$ setzen. Bilden wir nun die Zahlenkette $\left[-a_{0}, a_{1}\right]$, so beginnt sie mit $-a_{0}\left|a_{1}\right| a_{0}+a_{1}, a_{0}, a_{1} \ldots$ geht also nach 3 Gliedern in die Kette $\left[a_{0}, a_{1}\right]$ über. Ist nun $\frac{a_{0}}{a_{1}}=$ E $\{\alpha, \beta, \gamma, \ldots\}$, so ist also

$$
\frac{-a_{0}}{a_{1}}=\mathfrak{E}\{1,1, \alpha+1, \beta, \gamma, \ldots\} \text {. }
$$

Vom dritten Näherungsbruch an sind alle Zähler negativ.

Dagegen beginnt die Kette $\left[a_{0},-a_{1}\right]$ mit $a_{0},-a_{1}\left|a_{0}+a_{1}\right| a_{0}+2 a_{1}, a_{1}$ $a_{0}+a_{1}, a_{0}, a_{1} \ldots$, also ist

$$
\frac{a_{0}}{-a_{1}}=\mathbb{E}\{2,1,2, \alpha+1, \beta, \gamma, \ldots\} \text {. }
$$

Vom vierten Näherungsbruch an sind alle Nenner negativ.

Von diesen beiden $\mathfrak{E}$ న., die dieselbe Zahl darstellen, wollen wir nur den ersten als einen echten ER. ansehen. Ebenso können wir aber auch neben dem $\mathfrak{E} \Re$ für die positive rationale Zahl $\frac{a_{0}}{a_{1}}$ einen zweiten aufstellen, wenn wir $\frac{a_{0}}{a_{1}}=\frac{-a_{0}}{-a_{1}}$ setzen, also die Kette $\left[-a_{0},-a_{1}\right]$ bilden. Wir haben dabei zwei Fälle zu unterscheiden. Ist $a_{0}>a_{1}$, so beginnt die Kette mit $-a_{0}\left|-a_{1}\right| a_{0}-a_{1} \mid a_{0}, a_{1}, \ldots$, also ist

$$
\frac{-a_{0}}{-a_{1}}=\mathfrak{E}\{1,1,1, \alpha, \beta, \gamma, \ldots\} \text {. }
$$

Ist aber $a_{0}<a_{1}$, so hat der $\mathfrak{E} \Omega$. für $\frac{a_{0}}{a_{1}}$ die Form $\frac{a_{0}}{a_{1}}=\mathfrak{E}\{1, \alpha, \beta, \gamma, \ldots\}$ und die Kette $\left[-a_{0},-a_{1}\right]$ beginnt mit $-a_{0},-a_{1}\left|a_{1}-a_{0}\right| 2 a_{1}-a_{0}, a_{1}, \ldots$ Man erhält also den zugehörigen EN., indem man dem E্র. für $\frac{2 a_{1}-a_{0}}{a_{1}}$ $=2-\frac{a_{0}}{a_{1}}$ die Abschnitte 2,1 vorsetzt. Es ist aber offenbar $2-\frac{a_{0}}{a_{1}}$ $=\mathfrak{E}\{\alpha+1, \beta, \gamma, \ldots\}$, folglich

$$
\frac{-a_{0}}{-a_{1}}=\mathfrak{\&}\{2,1, \alpha+1, \beta, \gamma, \ldots\} \text {. }
$$


Auch die beiden letzten Formen wollen wir von der Betrachtung ausschließen; es ist also bei einem eigentlichen ER. entweder kein Abschnitt $=1$ (wenn der Wert des $\mathfrak{E} \Omega$. $a>1$ ) oder nur der erste Abschnitt (wenn $0<a<1$ ) oder die beiden ersten Abschnitte (wenn $a<0$ ist).

\section{§ 3. Unendliche Elementarkettenbrüche.}

Anstatt aus der Zahlenkette kann man einen Elementarkettenbruch auch durch den folgenden einfachen Algorithmus erhalten. Es sei $\omega$ eine reelle Zahl. Man bilde nacheinander die Zahlen

$$
\omega_{1}=\frac{1}{|\omega-1|}, \omega_{2}=\frac{1}{\left|\omega_{1}-1\right|}, \omega_{3}=\frac{1}{\left|\omega_{2}-1\right|}, \ldots
$$

Ist nun $\omega$ positiv, so folgt in der Reihe der Zahlen $\omega, \omega_{1}, \omega_{2}, \ldots$ stets auf eine Zahl, die $<1$ ist, eine Zahl $>1$. Man kann also die Reihe in Abschnitte von $\alpha, \beta, \gamma, \ldots$ Zahlen

$$
\begin{array}{lll}
\omega, & \omega_{1}, \quad \omega_{2}, \ldots & \omega_{a-1}, \\
\omega_{\alpha}, \quad \omega_{a+1}, \omega_{a+2}, \ldots & \omega_{\alpha+\beta-1}, \\
\omega_{\alpha+\beta}, \ldots & & \omega_{\alpha+\beta+\gamma-1},
\end{array}
$$

zerlegen, sodaß jeder Abschnitt mit einer Zahl $<1$ schließt, während alle übrigen Zahlen $>1$ sind. Mit diesen Zahlen bilde man den $\in$ S.

$$
\text { E }\{\alpha, \beta, \gamma, \ldots\} \text {, }
$$

und in diesem sind sicher alle Abschnitte vom zweiten $a b>1$.

Ist aber $\omega$ negativ, so ist $\omega_{1}=\frac{1}{1-\omega}<1$, es folgen also hier zwei Zahlen $<1$ auf einander, sie bilden jede für sich einen Abschnitt und der zugehörige ㅌ. hat die Form

$$
\leftleftarrows\{1,1, \gamma, \delta, \ldots\},
$$

worin alle Abschnitte vom dritten $a b>1$ sind.

Wir beweisen nun den Satz:

1. Ist $\omega$ eine rationale Zahl, so gelangt man in der Reihe der Zahlen $\omega, \omega_{1}, \omega_{2}, \ldots$ schließlich zu zwei aufeinanderfolgenden Zahlen $\omega_{l_{2}}=2, \omega_{l-1}=1$.

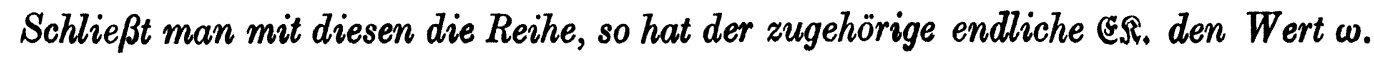


Beweis. Ist $\omega$ positiv und gleich dem Quotienten $\frac{a_{0}}{a_{1}}$ von teilerfremden ganzen Zahlen, so ist

$$
\omega_{1}=\frac{a_{1}}{\left|a_{0}-a_{1}\right|}=\frac{a_{1}}{a_{2}}, \omega_{2}=\frac{a_{2}}{\left|a_{1}-a_{2}\right|}=\frac{a_{2}}{a_{3}}, \ldots
$$

also bilden $a_{0}, a_{1}, a_{2}, a_{3}, \ldots$ eine Zahlenkette $\left[a_{0}, a_{1}\right]$. Diese schließt mit $a_{l-2}=2, a_{l-1}=1$, also wird $\omega_{l-2}=\frac{a_{l-2}}{a_{l-1}}=2$ und $\omega_{l-1}=\frac{1}{\omega_{l-2}-1}=1$. Die Gliederung der Kette $\left[a_{0}, a_{1}\right]$ stimmt offenbar mit der durch die Zahlen $\omega, \omega_{1}, \omega_{2}, \ldots$ erzeugten Gliederung $\{\alpha, \beta, \gamma, \ldots\}$ überein, folglich hat der $\mathbb{E} \Omega$. (3.) den Wert $\frac{a_{0}}{a_{1}}=\omega$.

Ist $\omega$ negativ, so ist $\omega_{1}=\frac{1}{1-\omega}<1$, also gleich dem endlichen $\Subset \Omega$. $\omega_{1}=\Subset\{1, \gamma, \delta, \ldots\}$. Dann ist aber $\omega=1-\frac{1}{\omega_{1}}$ gleich dem Eన. (4.). Damit ist der Satz bewiesen. Die Zahlen $\omega, \omega_{1}, \omega_{2}, \ldots$ sind die vollständigen Quotienten des ER.

Ist nun $\omega$ eine irrationale Zahl, so bricht die Reihe der Zahlen $\omega, \omega_{1}$, $\omega_{2}, \ldots$ nicht ab und der zugehörige ES. wird unendlich.

Ein unendlicher ES. heiße konvergent, wenn seine eigentlichen Näherungsbrüche gegen einen endlichen Grenzwert konvergieren.

Zur Untersuchung der Konvergenz eines unendlichen $\Subset \Omega$. betrachten wir die Differenzen von zwei Näherungsbrüchen $\frac{P_{i+x}}{Q_{i+x}}-\frac{P_{i}}{Q_{i}}$ für $\varkappa=1,2,3, \ldots$ Es ist nach (8.) und $\left(8^{\mathrm{a}}.\right)$ in $\S 3$ :

$$
\left|\frac{P_{i+x}}{Q_{i+x}}-\frac{P_{i}}{Q_{i}}\right|=\left|\frac{P_{i} x_{i x} \mp P_{i-1}}{Q_{i} x_{i x} \mp Q_{i-1}}-\frac{P_{i}}{Q_{i}}\right|=\frac{1}{Q_{i}\left(Q_{i} x_{i x} \mp Q_{i-1}\right)},
$$

wobei das obere oder untere Vorzeichen zu nehmen ist, je nachdem $\frac{P_{i}}{Q_{i}}$ ein Näherungsbruch am Schluß eines Abschnitts ist oder nicht. Im ersten Falle ist immer $x_{i x}>1$, also, da $Q_{i}-Q_{i-1}=Q_{i-2}$ ist:

$$
\left|\frac{P_{i+x}}{Q_{i+x}}-\frac{P_{i}}{Q_{i}}\right|<\frac{1}{Q_{i} Q_{i-2}}
$$

im zweiten Fall ist $x_{i x}>0$, also 


$$
\left|\frac{P_{i+x}}{\mid Q_{i+x}}-\frac{P_{i}}{Q_{i}}\right|<\frac{1}{Q_{i} Q_{i-1}} .
$$

Es ist also der unendliche $\mathfrak{A} \Omega$. jedenfalls konvergent, sobald die Nenner $Q_{i}$ mit $i$ über alle Grenzen wachsen. Das ist aber stets der Fall mit der einen Ausnahme, da $\beta$ die Nenner $Q_{1}, Q_{2}, Q_{3}, \ldots$ die periodische Zahlenkette 1, 1, 0, 1, 1,0,.. bilden. Dies tritt nur bei zwei Elementarkettenbrüchen ein, nämlich $\mathfrak{E}\{2,3,3,3, \ldots\}$ und $\mathfrak{E}\{1,1,3,3,3, \ldots\}$, und diese sind in der Tat divergent; die Näherungsbrüche des ersten gehen gegen $+\infty$, die des zweiten gegen $-\infty$. Es gilt also der Satz:

Ein unendlicher ER. ist - abgesehen von den beiden erwähnten Ausnahmen - stets konvergent. Man sieht leicht, $\mathrm{da} ß$ für eine endliche Irrationalzahl $\omega$ der Algorithmus (1.) niemals auf die Gliederung $\{2,3,3,3, \ldots\}$ oder $\{1,1,3,3,3, \ldots\}$ führen kann, also folgt:

3. Der einer Irrationalzahl $\omega$ zugeordnete unendliche $(\& \Omega$. (3.) oder (4.) ist stets konvergent und hat den Wert $\omega$.

Das letztere ergibt sich daraus, daß nach (1.) sich $\omega$ mit Hilfe der Näherungsbrüche des zugeordneten $\leftleftarrows \Re$. durch die Ausdrücke $\omega=$ $\frac{P_{i} \omega_{i} \mp P_{i-1}}{Q_{i} \omega_{i} \mp Q_{i-1}}$ darstellt und infolgedessen $\left|\omega-\frac{P_{i}}{Q_{i}}\right|$ gegen Null konvergiert.

Ein unendlicher ES. kann einen rationalen Wert haben. Man kann nämlich auch für ein rationales $\omega$ den Algorithmus (1.) unbegrenzt fortsetzen. An den Wert $\omega_{l-1}=1$ schließt sich an:

$$
\omega_{l}=\infty, \omega_{l+1}=0, \omega_{l+2}=1, \omega_{l+3}=\infty, \omega_{l+4}=0, \ldots
$$

Ist nun

$$
\omega=\mathfrak{E}\{\alpha, \beta, \ldots \sigma, \tau\}
$$

der endliche $\mathfrak{E} \Omega$. für $\omega$, so kann man entweder durch $\omega_{l}=\infty$ und $\omega_{l+1}=0$ den letzten Abschnitt um zwei vermehren, und dann folgen unbegrenzt viele Abschnitte gleich 3, oder man hat durch $\omega_{l}$ und $\omega_{l+1}$ einen neuen Abschnitt = 2 und darauf lauter Abschnitte $=3$, sodaß man für $\omega$ zwei unendliche Elementarkettenbrüche erhält:

$$
\omega=\mathfrak{E}\{\alpha, \beta, \ldots \sigma, \tau+2,3,3,3, \ldots\}
$$

und

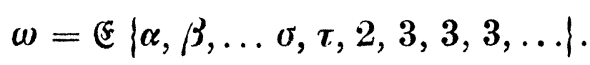


Beide ergeben in der Tat denselben Wert, wie der E્న. (5.). Sei nämlich $\omega=\frac{a_{0}}{a_{1}}$ der Wert dieses $\& \Omega$., $\frac{b_{0}}{b_{1}}$ sein vorletzter Näherungsbruch, so folgen beim $\mathfrak{E} \Omega$. (6.) auf $\frac{a_{0}}{a_{1}}$ die Näherungsbrüche

$$
\frac{a_{0}+b_{0}}{a_{1}+b_{1}}, \frac{2 a_{0}+b_{0}}{2 a_{1}+b_{1}} ; \frac{a_{0}}{a_{1}}, \frac{3 a_{0}+b_{0}}{3 a_{1}+b_{1}}, \frac{4 a_{0}+b_{0}}{4 a_{1}+b_{1}} ; \frac{a_{0}}{a_{1}}, \frac{5 a_{0}+b_{0}}{5 a_{1}+b_{1}}, \frac{6 a_{0}+b_{0}}{6 a_{1}+b_{1}} ; \ldots
$$

also ist der Wert des unendlichen $\mathfrak{E}$. (6.):

$$
\lim _{n=\infty} \frac{n a_{0}+b_{0}}{n a_{1}+b_{1}}=\frac{a_{0}}{a_{1}}=\omega
$$

Im Eミ. (7.) dagegen folgen auf $\frac{b_{0}}{b_{1}}, \frac{a_{0}}{a_{1}}$ die Näherungsbrüche

$$
\frac{a_{0}-b_{0}}{a_{1}-b_{1}}, \frac{2 a_{0}-b_{0}}{2 a_{1}-b_{1}} ; \frac{a_{0}}{a_{1}}, \frac{3 a_{0}-b_{0}}{3 a_{1}-b_{1}}, \frac{4 a_{0}-b_{0}}{4 a_{1}-b_{1}} ; \frac{a_{0}}{a_{1}}, \frac{5 a_{0}-b_{0}}{5 a_{1}-b_{1}}, \frac{6 a_{0}-b_{0}}{6 a_{1}-b_{1}} ; \ldots,
$$

also ist auch hier der Wert des $\mathfrak{E} \Omega$.

$$
\lim _{n \rightarrow \infty} \frac{n a_{0}-b_{0}}{n a_{1}-b_{1}}=\frac{a_{0}}{a_{1}}=\omega .
$$

Es kann also ein endlicher $\mathfrak{R}$. auf zwei Arten in einen unendlichen $\mathbb{E} \Omega$. verwandelt werden. Umgekehrt aber gilt der Satz:

4. Ein unendlicher $\mathfrak{R}$. hat dann und nur dann einen rationalen Wert, wenn von einer bestimmten Stelle an alle Abschnitte gleich 3 sind.

Es muß nämlich, wenn $\omega=\frac{a_{0}}{a_{1}}$ ist, der Ausdruck

$$
\left|Q_{i} \omega-P_{i}\right|=\frac{\left|Q_{i} a_{0}-P_{i} a_{1}\right|}{a_{1}}
$$

verschwinden, sobald er unter $\frac{1}{a_{1}}$ sinkt. Ist nun $\frac{P_{i}}{Q_{i}}$ nicht der letzte Näherungsbruch in einem Abschnitt, so ist

$$
\left|Q_{i} \omega-P_{i}\right|=\frac{1}{Q_{i} \omega_{i}+Q_{i-1}}
$$

also muß jedesmal, wenn $Q_{i} \omega_{i}+Q_{i-1}>a_{1}$ wird, $\omega_{i}=\infty$ sein. Der zu $\frac{P_{i}}{Q_{i}}$ gehörige vollständige Quotient ist also der oben gefundene divergente unendliche Eে $\omega_{i}=\mathfrak{E}\{2,3,3,3, \ldots\}$, folglich hat dann der E\&. für $\omega$ in 
der Tat die Form (6.). Ist aber $\frac{P_{i}}{Q_{i}}$ der letzte Näherungsbruch in einem $\mathrm{Ab}$ schnitt, so ist, da $Q_{i}=Q_{i-1}+Q_{i-2}$ ist:

$$
\left|Q_{i} \omega-P_{i}\right|=\frac{1}{Q_{i} \omega_{i}-Q_{i-1}}=\frac{1}{Q_{i-1}\left(\omega_{i}-1\right)+Q_{i-2} \omega_{i}}
$$

und auch hier ergibt sich, sobald der Nenner $>a_{1}$ wird, $\omega_{i}=\infty$. Dies führt aber zu einem unendlichen E̊. von der Form (7.).

Schließen wir also diese $E$. mit der „Periode 3 “ von der Betrachtung aus, womit auch die beiden divergenten $\Subset \Re$. ausgeschlossen sind, und nennen wir einen unendlichen $\&$., dessen Abschnitte nicht schließlich alle $=\mathbf{3}$ sind, regulär, so gilt der Satz:

5. Ein regulärer $\mathfrak{E}$ న. ist stets konvergent und stellt eine irrationale Zahl vor. Umgekehrt läßt sich eine irrationale Zahl immer nur auf eine Weise in einen unendlichen ㅌ. verwandeln, und dieser ist stets regulär.

Bei einem unendlichen $\mathbb{E} \Omega$. von endlicher Ordnung, also mit einer endlichen Anzahl von Abschnitten, ist der letzte Abschnitt unendlich lang, d. h. der Eన. schließt mit dem gewöhnlichen Kettenbruch $[1,1,1, \ldots]$, der als $\mathbb{E} \Re$. mit $\mathbb{E}\{\infty\}$ zu bezeichnen ist. Sein Wert, den wir mit $t$ bezeichnen wollen, ist

$$
t=\mathbb{E}|\infty|=\frac{\sqrt{5}+1}{2} .
$$

Es ist also ein unendlicher $\mathbb{E} \Re$. von endlicher Ordnung

$$
\mathfrak{E}\{\alpha, \beta, \ldots \tau, \infty)=\frac{a_{0} t-b_{0}}{a_{1} t-b_{1}},
$$

worin $\frac{b_{0}}{b_{1}}, \frac{a_{0}}{a_{1}}$ die beiden letzten Näherungsbrüche des endlichen $\mathbb{\Omega}$. $\mathbb{E}\{\alpha, \beta, \ldots \tau\}$ bezeichnen, und es besteht der Satz:

6. Jeder unendliche $\Subset \nwarrow$. von endlicher Ordnung ist arithmetisch äquivalent mit der quadratischen Irrationalzahl $t$.

Die Umkehrung dieses Satzes kann hier noch nicht in präziser Weise formuliert werden (vgl. § 5, Satz 11.).

Ein Abschnitt der durch den Algorithmus (1.) bestimmten Zahlen 
$\omega_{i}$ bestehe aus den Zahlen $\omega^{(1)}, \omega^{(2)}, \ldots \omega^{(\nu)}$, sodaß also $\omega^{(\nu)}<1$, die übrigen Zahlen $>1$ sind. Es ist dann

$$
\text { (9.) } \quad \omega^{(\nu-1)}=1+\frac{1}{\omega^{(\nu)}} ; \omega^{(\nu-2)}=1+\frac{1}{\omega^{(\nu-1)}} ; \ldots \omega^{(1)}=1+\frac{1}{\omega^{(2)}} .
$$

Damit $\omega^{(\nu)}$ in das Intervall $0<\omega^{(\nu)}<1$ hineinfällt, muß jede der vorhergehenden Zahlen in einem bestimmten Intervall liegen. Diese Intervalle sind alle voneinander getrennt und sind in folgender Weise festgelegt:

Es seien

$$
p_{0}=0, p_{1}=1, p_{2}=1, p_{3}=2, p_{4}=3, p_{5}=5, p_{6}=8, \ldots
$$

die bekannten Fibonaccischen Zahlen mit dem Bildungsgesetz

$$
p_{n}=p_{n-1}+p_{n-2} \text {. }
$$

Ihre Verhältnisse $\frac{p_{1}}{p_{0}}, \frac{p_{2}}{p_{1}}, \frac{p_{3}}{p_{2}}, \ldots$ sind die Näherungsbrüche des eben erwähnten Kettenbruchs \& $\{\infty\}=t=\frac{\sqrt{5}+1}{2}$, und sie begrenzen Intervalle $I_{1}, I_{2}, I_{3}, \ldots$, welche immer kleiner werdend von beiden Seiten gegen den Grenzpunkt $t$ konvergieren. Das $m^{\text {to }}$ Intervall ist

$$
I_{m}=\left(\frac{p_{m}}{p_{m-1}}, \frac{p_{m+2}}{p_{m+1}}\right) \text {. }
$$

Hierunter ist mit $p_{-1}=1$ auch das Intervall $(0,1)$ als $I_{0}$ einbegriffen.

Aus den Formeln (9.) folgt dann der Satz:

7. Damit $0<\omega^{(\nu)}<1$ ist, müssen die Zahlen $\omega^{(\nu-m)}$ für $m=1,2,3, \ldots$ jeweils im Intervall $I_{m}$ liegen.

Oder anders ausgedrückt:

8. Liegt eine Zahl $\omega_{i}$ im Intervall $I_{m}$, so folgen auf sie im gleichen Abschnitt noch $m$ Zahlen, welche der Reihe nach in den Intervallen $I_{m-1}$, $I_{m-2}, \ldots I_{1}, I_{0}$ liegen.

\section{§ 4. Elementarkettenbrüche und lineare Substitutionen.}

Es sei $\frac{P_{i}}{Q_{i}}=\mathfrak{E}\{\alpha, \beta, \ldots \mu\}$ ein Näherungsbruch der $\mathbb{E}_{\Omega_{.}}$-Entwicklung einer Größe $x$; der zugehörige vollständige Quotient sei $y$, also

$$
x=\frac{P_{i} y \mp P_{i-1}}{Q_{i} y \mp Q_{i-1}}
$$


je nachdem $\frac{P_{i}}{Q_{i}}$ der Näherungsbruch am Schluß eines Abschnitts ist oder nicht. Wir schreiben dann den E̊. für $x$ im einen oder anderen Fall in der Form

$$
x=\Subset\{\alpha, \beta, \ldots \lambda, \mu \| y\}
$$

oder

$$
x=\mathfrak{E}\{\alpha, \beta, \ldots \lambda, \mu \mid y\} \text {. }
$$

Die Zahlen $x$ und $y$ sind durch eine ganzzahlige Substitution von der Determinante \pm 1 mit einander verknüpft. Diese läßt sich aus zwei Fundamentalsubstitutionen $x^{\prime}=1+\frac{1}{x}$ und $x^{\prime}=1-\frac{1}{x}$ oder

$$
U=\left(\begin{array}{ll}
1 & 1 \\
1 & 0
\end{array}\right), \quad \nabla=\left(\begin{array}{rr}
1 & -1 \\
1 & 0
\end{array}\right)
$$

zusammensetzen, und die Zusammensetzung ist durch den $\mathbb{E} \Re$., unmittelbar gegeben. Es ist nämlich auf Grund der Bildungsweise der E尺., je nachdem zwischen $x$ und $y$ die Beziehung (2.) oder (3.) besteht:

$$
\begin{aligned}
& x=U^{\alpha-1} V U^{\beta-1} V \ldots U^{\mu-1} V(y) \text { oder } \\
& x=U^{\alpha-1} V U^{\beta-1} V \ldots U^{\mu}(y) .
\end{aligned}
$$

Wir nennen eine Substitution*) $\left(\begin{array}{ll}\alpha & \beta \\ \gamma & \delta\end{array}\right)$, die sich allein aus den Substitutionen $U$ und $V$ zusammensetzt, eine gerade Substitution und haben den Satz:

1. Der Wert des ER. ist mit irgend einem vollständigen Quotienten durch eine gerade Substitution verknüpft.

Für die Fundamentalsubstitutionen $U$ und $V$ gelten die Beziehungen $\left.{ }^{* *}\right)$ :

$$
\begin{gathered}
V^{8}=1, \text { also } V^{-1}=V^{2} \\
U V^{2} U V^{2}=1, \text { also }
\end{gathered}
$$

*) Eine Verwechslung der Substitutionskoeffizienten $\alpha, \beta$ mit den gleich bezeichneten Abschnitten des CR. ist wohl nicht zu befürchten.

**) Dabei werden $\left(\begin{array}{ll}\alpha & \beta \\ \gamma & \delta\end{array}\right)$ und $\left(\begin{array}{ll}-\alpha & -\beta \\ -\gamma & -\delta\end{array}\right)$ als identisch angesehen. 


$$
V=U V^{2} U, U^{-1}=V^{2} U V^{2} .
$$

Man sieht ohne weiteres:

2. Die geraden Substitutionen bilden eine Gruppe.

Infolge von (7.) und (8.) kann jede gerade Substitution auf eine solche Form gebracht werden, daß sie die Potenzen von $U$ nur mit positiven Exponenten und zwischen ihnen nur die erste Potenz von $V$ enthält, d. h. sie läßt sich stets auf eine der Formen (5.) oder (6.) reduzieren. Darin kann $\alpha=1$ oder gleichzeitig $\alpha=1, \beta=1$ sein; dann beginnen die Substitutionen mit $V$ oder $V^{2}$. Ferner kann in (5.) auch $\mu=1$ sein; dann schließt die Substitution mit $V^{2}$. Die übrigen Zahlen $\gamma, \delta, \ldots \lambda$ sind sämtlich größer als 1 . Dies entspricht den früheren Festsetzungen über die Elementarkettenbrüche, wonach nur die beiden ersten Abschnitte und der letzte Abschnitt gleich 1 sein sollen. Solche ER. wollen wir jetzt normal nennen. Wir können nun aber auch ㅌ․ betrachten, bei denen einzelne innere Abschnitte gleich 1 sind, und können sie, indem wir sie mit den linearen Substitutionen in Beziehung setzen, in normale $\mathbb{E} \Re$. verwandeln. Es mögen also im $\mathfrak{A}$. die Abschnitte $\varkappa, 1, \lambda$ aufeinanderfolgen, wobei $*$ und $\lambda>1$ sind. Ihnen entspricht in der zugeordneten Substitution das Produkt $U^{x-1} V V U^{\lambda-1} V$ oder nach (8.): $U^{x-2} V U^{\lambda-2} V$, folglich ist

(9.) $\mathbb{E}\{\alpha, \beta, \ldots \varkappa, 1, \lambda, \ldots\}=\mathbb{E}\{\alpha, \beta, \ldots \varkappa-1, \lambda-1, \ldots\}$.

Sind aber zwei aufeinanderfolgende innere Abschnitte gleich 1, so entspricht den Abschnitten $\varkappa, 1,1, \lambda$ das Produkt $U^{x-1} V V^{2} U^{\lambda-1} V=U^{x+\lambda-2} V$, also ist

$$
\mathfrak{E}\{\alpha, \beta, \ldots \%, 1,1, \lambda, \ldots\}=\mathfrak{E}\{\alpha, \beta, \ldots \varkappa+\lambda-1, \ldots\} .
$$

Sind drei aufeinanderfolgende innere Abschnitte gleich 1, so entspricht dies dem Produkt $U^{x-1} V V^{3} U^{\lambda-1} V=U^{x-1} V U^{\lambda-1} V$, d. h. es ist

(11.) $\mathbb{E}\{\alpha, \beta, \ldots *, 1,1,1, \lambda, \ldots\}=\mathbb{E}\{\alpha, \beta, \ldots \varkappa, \lambda, \ldots\}$.

Auf diese drei Fälle läßt sich die Reduktion aller nicht normalen ङS. zurückführen. So ist z. B. nach (9.) und (11.)

$$
\mathfrak{E}\{3,1,2,1,1,4\}=\mathfrak{E}\{2,1,1,1,4\}=\mathfrak{E}\{2,4\}
$$

oder nach (10.) und (9.)

$$
\mathfrak{E}\{3,1,2,1,1,4\}=\mathbb{E}\{3,1,5\}=\mathfrak{E}\{2,4\} .
$$

Es seien nun $x$ und $x^{\prime}$ zwei irrationale Zahlen, deren $\mathbb{E} \Omega$. von einer Journal für Mathematik. Bd. 149. Heft 1/2. 
bestimmten Stelle an übereinstimmen, also einen vollständigen Quotienten $y$ gemeinsam haben. Dann folgt aus Satz 1 , daß $x$ und $x^{\prime}$ durch eine gerade Substitution verknüpft sind. $Z_{w}$ ei solche Zahlen nennen wir geradeäquivalent. Es besteht also der Satz:

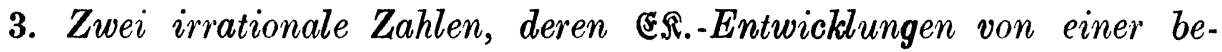
stimmten Stelle an übereinstimmen, sind gerade-äquivalent.

Es gilt aber auch die Umkehrung dieses Satzes. Sind $x$ und $x^{\prime}$ gerade-äquivalent, so ist entweder

(12.) $x^{\prime}=U^{\alpha-1} V U^{\beta-1} V \ldots U^{\mu-1} V(x)=\Subset\{\alpha, \beta, \ldots \mu \| x\}$

oder

(12a $\left.{ }^{\mathrm{a}}\right) \quad x^{\prime}=U^{\alpha-1} V U^{\beta-1} V \ldots U^{\mu}(x)=\mathcal{F}\{\alpha, \beta, \ldots \mu \mid x\} ;$

wir haben also den Satz:

4. Sind zwei Zahlen gerade-äquivalent, so stimmen ihre \&્..-Entwicklungen von einer bestimmten Stelle an überein.

Es kann dabei stets erreicht werden, daß die eine Zahl ein vollständiger Quotient in der Eאß.-Entwicklung der anderen Zahl ist.

Es seien nun $x$ und $x^{\prime}$ irgendwie äquivalent, d. h. es sei

$$
x^{\prime}=\frac{\alpha x+\beta}{\gamma x+\delta}
$$

mit vier ganzen Zahlen $\alpha, \beta, \gamma, \delta$ von der Determinante $\varepsilon= \pm 1$. In der Entwicklung von $x$ in einen normalen $\mathbb{S}^{\Omega}$. sei $\frac{P_{i}}{Q_{i}}$ ein Näherungsbruch am Schluß eines Abschnitts, $y$ der zugehörige vollständige Quotient, dann ist

$$
\begin{gathered}
x=\frac{P_{i} y-P_{i-1}}{Q_{i} y-Q_{i-1}} \text { und } \\
P_{i} Q_{i-1}-P_{i-1} Q_{i}=\partial_{i}= \pm 1 .
\end{gathered}
$$

Dies in (13.) eingeführt gibt

$$
\begin{gathered}
x^{\prime}=\frac{A y-B}{C y-\bar{D}}, \text { worin } \\
A=\alpha P_{i}+\beta Q_{i}, \quad B=\alpha P_{i-1}+\beta Q_{i-1}, \\
C=\gamma P_{i}+\delta Q_{i}, \quad D=\gamma P_{i-1}+\delta Q_{i-1},
\end{gathered}
$$

und es ist 


$$
A D-B C=\varepsilon \partial_{i}= \pm 1 \text {. }
$$

Es seien nun zunächst $x$ und $x^{\prime}$ positiv und die Vorzeichen von $\alpha$, $\beta, \gamma, \delta$ so gewählt, daß $\alpha x+\beta$, also auch $\gamma x+\delta$ positiv ist. Dann werden bei genügend großem $i$ die Zahlen $A, B, C, D$ positiv, und es wird

$$
A>B, \quad C>D \text {. }
$$

In dem Æ̊. für $\frac{A}{\bar{C}}$ sei $\frac{B^{\prime}}{\bar{D}^{\prime}}$ der vorletzte Näherungsbruch. Dann können zwei Fälle eintreten:

1. Es ist

$$
A D^{\prime}-B^{\prime} C=A D-B C,
$$

und dann folgt in bekannter Weise, daß

$$
B^{\prime}=B, \quad D^{\prime}=D
$$

sein muß, also ist nach (14.) $y$ auch ein vollständiger Quotient der E爪.Entwicklung von $x^{\prime}$, folglich sind $x$ und $x^{\prime}$ gerade-äquivalent.

2. Es ist

$$
\begin{gathered}
A D^{\prime}-B^{\prime} C=-A D+B C, \text { also } \\
A\left(D+D^{\prime}\right)=C\left(B+B^{\prime}\right),
\end{gathered}
$$

und hieraus folgt, da $B+B^{\prime}<2 A, D+D^{\prime}<2 C$ ist, da $B$

$$
B+B^{\prime}=A, D+D^{\prime}=C
$$

sein muß. Es ist dann also $\frac{B}{D}$ der dem Bruch $\frac{B^{\prime}}{\bar{D}^{\prime}}$ vorangehende Näherungs-

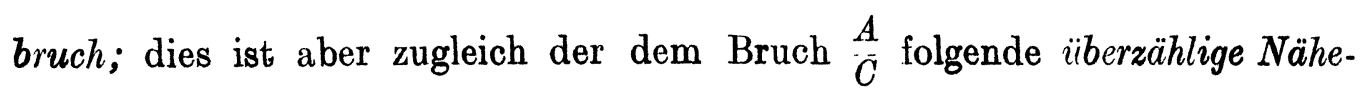
rungsbruch, $\mathrm{d}$. h. ist

$$
\frac{A}{C}=\Subset\{a, b, \ldots n\},
$$

so ist

$$
\bar{B}=\mathfrak{D}\{a, b, \ldots n, 1\} \text {. }
$$

Nach (14.) ist dann

$$
x^{\prime}=\frac{B \cdot \frac{1}{y}-A}{D \cdot \frac{1}{y}-C},
$$


und daraus schließt man, daß in der ER.-Entwicklung von $x^{\prime}$ der Bruch $\bar{D}$ ein Näherungsbruch am Schluß eines Abschnitts und $\frac{1}{y}$ der zugehörige vollständige Quotient ist. Wir nennen in diesem Fall $x$ und $x^{\prime}$ ungeradeäquivalent.

Ist

$$
y=\mathfrak{E}\{\nu, \pi, \rho, \sigma, \ldots\},
$$

so hat der reziproke $\mathbb{E}\{$., da $\nu>1$ ist, die Form

$$
\frac{1}{y}=\mathfrak{E}\left\{1, \nu^{\prime}, \pi^{\prime}, \varrho^{\prime}, \ldots\right\},
$$

also wird

oder nach (10.)

$$
x^{\prime}=\mathbb{E}\left\{a, b, \ldots n, \mathbf{1}, \mathbf{1}, \boldsymbol{v}^{\prime}, \boldsymbol{\pi}^{\prime}, \boldsymbol{\varphi}^{\prime}, \ldots\right\}
$$

$$
x^{\prime}=\mathfrak{E}\left\{a, b, \ldots n+v^{\prime}-1, \pi^{\prime}, \varrho^{\prime}, \ldots\right\} .
$$

Es besteht also der Satz:

5. Sind zwei Zahlen ungerade-äquivalent, so sind ihre ER. - Entwicklungen von einer bestimmten Stelle an zueinander reziprok.

Der Satz bleibt bestehen, wenn eine oder beide Zahlen negativ sind, denn es ist $-x$ zu $x$ gerade-äquivalent vermöge der Substitution $V^{2} U$, sodaß $-x=\mathfrak{E}\{1,1,1 \mid x\}$. Ist nun z. B. nur $x$ negativ, so gilt der Satz für $-x$ und $x^{\prime}$, folglich auch für $x$ und $x^{\prime}$.

Zwei ungerade-äquivalente Zahlen sind durch eine ungerade Substitution miteinander verknüpft. Es ist

$$
\begin{aligned}
& x=\Subset\{\alpha, \beta, \ldots \mu \| y\}, \\
& x^{\prime}=\Subset\left\{a, b, \ldots m \| \frac{1}{y}\right\},
\end{aligned}
$$

also, wenn wir als neue Fundamentalsubstitution die reziproke Transformation

$$
R=\left(\begin{array}{ll}
0 & 1 \\
1 & 0
\end{array}\right)
$$

einführen:

$$
\begin{aligned}
x & =U^{a-1} V U^{\beta-1} V \ldots U^{\mu-1} V(y), \\
x^{\prime} & =U^{a-1} V U^{b-1} V \ldots U^{m-1} V R(y) .
\end{aligned}
$$


Durch Umkehrung der ersten Substitution erhält man, wenn man zur Abkürzung

$$
V(U V)^{\alpha-1} V=T_{a}
$$

setzt:

und damit

$$
y=T_{\mu} T_{\lambda} \ldots T_{\beta} T_{\alpha}(x)
$$

$$
x^{\prime}=U^{a-1} V \ldots U^{m-1} V R T_{\mu} \ldots T_{a}(x),
$$

d. h. eine ungerade Substitution setzt sich aus den drei Substitutionen $U, V, R$ zusammen.

Die vorliegende Substitution läßt sich bedeutend vereinfachen. Für die Substitution $R$ gelten die Beziehungen

$$
\begin{gathered}
R^{2}=1, \text { also } R^{-1}=R \\
R U R=V U, R V R=V^{2}, R V^{2} R=V .
\end{gathered}
$$

Hieraus schließt man sofort den Satz:

6. Jede aus $U, V, R$ zusammengesetzte Substitution, die eine gerade Anzahl von Substitutionen $R$ enthält, läßt sich allein durch $U$ und $V$ ausdrücken, ist also eine gerade Substitution.

Es läßt sich also eine ungerade Substitution aus den Substitutionen $U, V$ und einer Substitution $R$ zusammensetzen, und man kann stets erreichen, daß die Substitution $R$ am Ende steht. Um (18.) in dieser Weise umzuformen, bilden wir mit Benutzung von (19.)

$$
R(U V) R=R U R R V R=V U V^{2},
$$

ferner

$$
R T_{\mu} R=R V R R(\stackrel{1}{U V}) R \ldots R\left(\stackrel{\frac{\mu-1}{U V}}{\bar{U}}\right) R R V R=U^{\mu-1} V
$$

folglich

$$
R T_{\mu} \ldots T_{\alpha}=U^{\mu-1} V U^{\lambda-1} V \ldots U^{\alpha-1} V R .
$$

Damit wird (18.):

$$
x^{\prime}=U^{a-1} V \ldots U^{m-1} V U^{\mu-1} V \ldots U^{a-1} V R(x) .
$$

Hieraus schließt man:

7. Haben zwei ungerade.äquivalente Zahlen die in (16.) angegebenen ङ尺.-Entwicklungen, so ist 


$$
x^{\prime}=\mathfrak{E}\left\{a, b, \ldots m, \mu, \lambda, \ldots \beta, \alpha \| \frac{1}{x}\right\}
$$

und umgekehrt, wie man leicht sieht:

$$
x=\mathfrak{E}\left\{\alpha, \beta, \ldots \mu, m, \ldots b, a \| \frac{1}{x^{\prime}}\right\} .
$$

Es ist nun auch sofort $\mathrm{zu}$ sehen, daß jede aus $U, V$ und einer Substitution $\boldsymbol{R}$ zusammengesetzte Substitution eine ungerade Substitution ist. Eine derartige Substitution läßt sich immer auf eine der beiden Formen

$$
\begin{aligned}
& x^{\prime}=U^{a-1} V \ldots U^{\mu-1} V R(x), \\
& x^{\prime}=U^{a-1} V \ldots U^{\mu} R(x)
\end{aligned}
$$

bringen. Diese entsprechen den ER.

$$
x^{\prime}=\mathfrak{E}\left\{\alpha, \beta, \ldots \mu \| \frac{1}{x}\right\}
$$

und

$$
\left.x^{\prime}=\Subset|\alpha, \beta, \ldots \mu| \frac{1}{x}\right\}=\Subset\left\{\alpha, \beta, \ldots \mu+1,1,1 \| \frac{1}{x}\right\},
$$

d. h. es sind in der Tat $x$ und $x^{\prime}$ ungerade-äquivalent.

Zusammenfassend können wir sagen:

8. Alle ganzzahligen linearen Substitutionen von der Determinante \pm 1 zerfallen in zwei Arten: Gerade Substitutionen, die sich allein a us den Fundamentalsubstitutionen $U$ und $V$, und ungerade Substitutionen, diesich aus $U, V$ und einer Substitution $R$ zusammensetzen. Die geraden Substitutionen bilden eine Gruppe, zwei ungerade Substitu tionengebenzusammengesetzteine gerade Substitution. Die ङ §. - Entwicklungen von zwei äquivalenten Zahlen stimmen entweder von einer bestimmten Stelle an überein oder sie sind von einer bestimmten Stelle an reziprok, je nachdem die Zahlen durcheine geradeoder ungerade Substitution miteinander verknüpft sind*).

*) Vgl. hierzu meine Arbeit „i:ber Möbiuskettenbrüche und Elementarkettenbrüche", Math. Zeitschr. Bd. 3. 
Bei dieser Einteilung der linearen Substitutionen in zwei Arten kommt die Scheidung in eigentliche (Determinante +1 ) und uneigentliche Substitutionen (Determinante - 1) nicht zum Ausdruck. Dies gelingt aber in sehr übersichtlicher Weise, wenn wir an Stelle von $U$ die Substitution $x^{\prime}=-x$ oder

$$
N=\left(\begin{array}{rr}
-1 & 0 \\
0 & 1
\end{array}\right)=V^{2} U
$$

und an Stelle von $R$ die Substitution $x^{\prime}=-\frac{1}{x}$ oder

$$
T=\left(\begin{array}{rr}
0 & 1 \\
-1 & 0
\end{array}\right)=N R
$$

einführen. Dann ist

$$
N^{2}=1, T^{2}=1, V^{3}=1,
$$

ferner

$$
\begin{gathered}
T N T=N, N T N=T, \\
N V N=T V^{2} T, N V^{2} N=T V T,
\end{gathered}
$$

und es besteht der Satz:

9. Alle ganzzahligen linearen Substitutionen von der Determinante \pm 1 lassen sich aus den drei zyklischen Substitutionen $N, V, T$ zusammensetzen. Jede Substitution mit einer geraden Anzahl von $T$ läßt sich durch $N$ und $V$, jede Substitution mit einer geraden Anzahl von $N$ läßt $\operatorname{sich} d u r c h$ $T$ und $V$ ausdrücken.

Dies führt in doppelter Weise zu einer Einteilung der linearen Substitutionen.

I. Gerade Substitutionen nur aus $V$ und $N$ zusammengesetzt.

Ungerade $\quad, \quad, \nabla, N$ und einer Substitution $T \mathrm{zu}$ sammengesetzt.

II. Eigentliche Substitutionen nur aus $V$ und $T$ zusammengesetzt.

Uneigentliche $\quad, \quad, \quad V, T$ und einer. Substitution $N$ zusammengesetzt.

Die Translation $x^{\prime}=x+1$ oder $S=\left(\begin{array}{ll}1 & 1 \\ 0 & 1\end{array}\right)$ ist eine ungerade Substitution, denn es ist

(21.)

$$
S=V T=U R
$$


Ein einfaches Kennzeichen für gerade Substitutionen hat man in dem folgenden Satz, auf den mich Herr $J$. Schur aufmerksam gemacht hat:

10. Die Gruppe der geraden Substitutionen stimmt überein mit der Gruppe der Substitutionen, die mod 2 einer der Substitutionen

kongruent sind.

$$
E=\left(\begin{array}{ll}
1 & 0 \\
0 & 1
\end{array}\right), V=\left(\begin{array}{rr}
1 & -1 \\
1 & 0
\end{array}\right), V^{2}=\left(\begin{array}{rr}
0 & 1 \\
-1 & 1
\end{array}\right)
$$

Ist nämlich $\mathfrak{B}$ die Gruppe der geraden Substitutionen, $\mathfrak{B}^{\prime}$ die in dem Satz genannte Gruppe, so ist $\mathfrak{B}$ in $\mathfrak{B}^{\prime}$ enthalten, da die Substitutionen $V$ und $N$ in $\mathfrak{B}^{\prime}$ enthalten sind. Daß beide Gruppen identisch sind, folgt einfach daraus, daß $\mathfrak{B}$ und $\mathfrak{B}^{\prime}$ Untergruppen vom Index 2 in der Gruppe aller ganzzahligen unimodularen Substitutionen sind.

Es ist also $\left(\begin{array}{ll}\alpha & \beta \\ \gamma & \delta\end{array}\right)$ eine gerade Substitution, wenn entweder $\beta$ und $\gamma$ gerade oder $\alpha, \beta, \gamma$ ungerade oder $\beta, \gamma ; \delta$ ungerade sind.

Zugleich ergibt sich, daß die ungeraden Substitutionen diejenigen sind, die $\bmod 2$ einer der Substitutionen

$$
T=\left(\begin{array}{rr}
0 & 1 \\
-1 & 0
\end{array}\right), T V=\left(\begin{array}{rr}
1 & 0 \\
-1 & 1
\end{array}\right), T V^{2}=\left(\begin{array}{lr}
1 & -1 \\
0 & 1
\end{array}\right)
$$

kongruent sind.

Aus Satz 10. schließt man sofort den folgenden Satz, dessen sehr einfache Begründung ich ebenfalls Herrn $J$. Schur verdanke.

11. Jede eigentliche Substitution von der Form $\left(\begin{array}{l}\alpha \\ \gamma-\alpha\end{array}\right)$ ist eine ungerade Substitution.

Es könnte nämlich die Substitution nur gerade sein, wenn $\beta$ und $\gamma$ gerade sind. Dann wäre aber $\alpha^{2}=-\beta \gamma-1 \equiv 3 \bmod 4$, was unmöglich ist. 